\title{
Culture as an ingredient of personalized medicine
}

\author{
Kimberly Matheson, PhD; Amy Bombay, PhD; Hymie Anisman, PhD
}

Personalized (precision) medicine approaches to deal with many mental and physical illnesses have often focused on biomarkers along with recognition of the importance of a constellation of developmental, psychosocial and contextual factors in relation to the development of illnesses as well as treatment efficacy. Acknowledgement of the breadth of health determinants has further enabled the application of a precision approach to public health interventions. ${ }^{1}$ This said, greater appreciation is needed concerning the contribution of cultural factors to mental illnesses. This need has become particularly evident to us in our work with indigenous populations.

Several approaches to the diagnosis and treatment of mental issues have been developed that focused primarily on the biomedical features of a precision medical model (e.g., endophenotypic analyses). However, incorporation of the interactive and direct effects of psychosocial determinants on neurobiological factors expanded the understanding of processes leading to illness and treatment of illness. In this regard, rather than focusing only on neurobiological factors, the National Institute of Mental Health Research Domain Criteria (RDoC) approach, which was developed as a framework for understanding mental illnesses and as a step toward personalized treatments, included multiple psychosocial factors in characterizing various conditions, ${ }^{2}$ but it was not met with complete unanimity. ${ }^{3,4}$ Kirmayer and $\mathrm{Craffa}^{5}$ were especially persuasive in their critique of the RDoC approach, indicating that it failed to consider cultural factors, including social norms and values, geographical conditions and environmental exposures, that could influence the development and course of disease as well as the effectiveness of treatments. For cultural groups who define wellness differently from Western medical science and who have different orientations toward healing practices, some of the variables considered in the RDoC might have limited value. For example, although Western approaches would lead us to anticipate significant rates of posttraumatic stress disorder following the trauma of ethnic genocide and civil war, trauma symptoms expressed among survivors vary considerably, and Western therapeutic treatments can be ineffectual if not counterproductive. ${ }^{6,7}$
Increasingly, cultural factors in mental health are becoming apparent through recognition of the ineffectiveness of applying Western assumptions to identification, etiology and treatment of mental disorders..$^{8,9}$

\section{Deeper implications of culture}

With a few exceptions, ${ }^{10}$ the deeper implications of cultural variations in association with biological processes have not been unpacked. Culture is not an essentialist or static construct. It changes over time (cultural evolution), and environmental conditions shape social structures and relationships, beliefs, activities, diet and physical adaptations. Moreover, culture is rooted in ancestry (systems of knowledge), historical events (collective trauma), and evolving contexts (climate change, colonization, migration). The recognition of the need for cultural relevance has evoked debates regarding local versus global orientations. Conversely, although culture is thought to reflect a social (sometimes even national) context, its significance varies across individuals. This includes variations in the extent to which individuals see themselves as embodying their cultural norms, their sense of belonging versus marginalization, and their feelings of pride and collective esteem versus shame, resentment, or anxiety. Such variations contribute to whether individuals turn to their cultural roots as a resource that enables resilience in the face of adversity, particularly through processes such as collective meaningmaking, social support seeking, or the mobilization of collective actions. Effectively, cultural specificities and variations shape a core part of individuals' identity in ways that can be integral to mental health.

\section{Limiting personalized medicine to Western cultural assumptions}

In our own work (and that of others) with Aboriginal peoples in Canada, the limitations of a personalized treatment approach that does not extend beyond Western cultural assumptions have become especially evident. In an editorial previously published in this journal, ${ }^{11}$ some of the mental

Correspondence to: H. Anisman, Department of Neuroscience, Carleton University, Ottawa ON K1S 5B6;

HymieAnisman@cunet.carleton.ca.

DOI: $10.1503 / j p n .170234$ 
health problems encountered by Aboriginal peoples in Canada were considered, including the high rates of depression, suicide, trauma and drug use, as were some steps to achieve mental wellness. In Canada (and internationally), the importance of culture and cultural safety has been highlighted by academics and indigenous organizations as being a key consideration in efforts to reduce the significant health inequities faced by Aboriginal peoples. This said, the notion of culture is especially difficult to incorporate when one takes into consideration the assimilationist policies that targeted Aboriginal peoples and that have had a serious impact on the transmission of culture (in all of its meanings) to current generations. Following many centuries of evolving indigenous cultures that were well adapted to their social and environmental contexts, the past few centuries have purposely and markedly altered the course of Aboriginal peoples' knowledge frameworks. The tides of indigenous cultures continue to shift, as many individuals and communities are reclaiming traditions in a manner that enables them to adapt and flourish in a contemporary world.

Since first contact with European settlers, Aboriginal peoples in Canada were exposed to diseases that decimated their population, followed by the dispossession of their land and relocation either by force or because settler resource development rendered the land unusable for hunting, fishing, or cultivation of natural plants. Indigenous identities and cultures were further eroded by colonizing policies (e.g., the Indian Act, Indian residential schools) that attempted forced assimilation and undermined the practice of traditional skills and relationships to the land. Continued systemic discrimination and identity disruptions undermined the mental health of individuals, families and communities, who lost their traditional culture and were alienated from modern society. Youth, in particular, have been negatively affected as they try to claim a positive cultural identity, often while in the throes of community dysfunction. This is further compounded by feelings of shame and diminished pride resulting from the internalization of racial stereotypes and prejudices. At the same time, Aboriginal Elders and adults willing to share their narratives of resistance about their collective trauma experiences may contribute to resilience and healing and might promote greater cultural awareness and pride among subsequent generations..$^{12,13}$

\section{Cultural, psychosocial, environmental and biological influences}

Although not often considered, superimposed on this sociocultural backdrop are the effects of climate change. Because a connection to the land has been central to indigenous cultures, for many northern communities the climate changes that have been occurring have served as a tipping point for yet greater mental health challenges. Elders, especially, are encountering environmental conditions that they are unable to predict or recognize. This loss of traditional knowledge has implications for intergenerational relationships, as Elders' acquired knowledge and wisdom no longer seem relevant. ${ }^{14}$ Moreover, many mental health interventions among Aboriginal peoples encourage (re)connection to the land as part of strengthening cultural identity and as a coping resource. Unfortunately, with shifts in climate and consequences for the health of the land and wildlife, this therapeutic strategy has the potential to become disheartening and confusing. ${ }^{15}$

With some variations, Aboriginal peoples in the United States, Australia, New Zealand and in regions of South America and Mexico have experienced comparable collective traumas over the past few centuries. Within these countries, there has been increasing recognition that Western assimilative practices have diminished rather than improved health conditions for indigenous populations. Efforts to address health inequities are being made, and though Western treatment approaches might bring about some improvements (e.g., trauma-focused cognitive behavioural therapy), particularly if adapted to be culturally appropriate (e.g., by adopting a "2-eyed seeing" approach to healing ${ }^{16}$ ), data regarding the effectiveness of such approaches among Aboriginal peoples are needed. Quantitative or biological data are sparse, as historically the collection of such data has been done in an exploitative manner that did not serve to benefit (and has often been to the detriment of) the wellbeing of Aboriginal peoples.

That said, there is a growing mutual appreciation that much would be gained by understanding the interplay between culture, psychosocial influences, environmental factors and biological mechanisms as well as how these associations shift over generations. In recent years, it has become evident that different cultures (typically defined in research by national origins or racial self-identification) exhibit varied profiles associated with genes and gene polymorphisms, both in terms of rates of expression and function. ${ }^{15}$ Epigenetic profiling has suggested the presence of populationspecific signatures correlating with phenotypic characteristics. ${ }^{17}$ Pursuing a better understanding of how culture can bring about such differences has implications for the identification of biomarkers predicting illnesses and may inform the likely efficacy of particular treatment regimens (psychosocial, behavioural, or pharmacological). ${ }^{18}$

Certainly, Aboriginal peoples have been exposed to multiple sequential and concurrent events that might trigger epigenetic changes. Collective and historical traumas (e.g., the Holocaust) have transgenerational effects, ${ }^{19}$ which conceivably might have biological and interpersonal consequences, and in the case of Aboriginal peoples could have actions on the effective functioning of whole communities. ${ }^{20}$ The multiple mechanisms (parenting, (over)communication or abject silence, collective storytelling, meaning-making and epigenetic changes) by which trauma is transmitted across generations continues to be a research focus. ${ }^{21}$ It should be underscored that although polymorphisms and epigenetic changes might render individuals at increased risk for pathology, from an evolutionary perspective, the genetic variations might also contribute to increased resilience in the face of adversity. In this regard, although some epigenetic changes are linked to collective historical trauma, Aboriginal peoples 
have also shown the capacity to persevere, and some communities and individuals have flourished despite historical trauma. Unfortunately, the data necessary to determine genetic and epigenetic markers that might be relevant to both the vulnerabilities and strengths and how they interact with cultural and psychosocial processes among indigenous populations do not currently exist. Yet, such understanding might be critical for the development of personalized treatment approaches that build on cultural strengths and mobilize existing psychosocial resources.

Evolutionary gene adaptations and the links to environmental factors that influence culture are gaining research attention. ${ }^{22}$ The physical environment, which is embedded in and shapes culture, can affect diet, activity levels and exposure to animals and wildlife. These factors influence the microbiome, which we are only beginning to understand in association with physical and mental health, although the available data suggest that microbiota alter immune and brain functioning and could thereby impact mental wellness. ${ }^{23}$ For instance, selection pressures that existed among Greenland Inuit may have resulted in a preponderance of particular gene mutations that limit diseases that would otherwise come with a diet rich in protein and omega-3 polyunsaturated fatty acids. These mutations, which are relatively rare in Europeans, modulate fatty acid composition, contribute to lowered low-density lipoproteins and fasting insulin levels, and serve in the regulation of growth hormones. ${ }^{24}$ These data support the view that culturally based personalized diets (much like personalized medicine) might be best for determining the foods that are healthy for any given individual. These, in turn, could influence microbial and inflammatory immune processes that affect mental and physical well-being.

While evolutionary changes can lead to increased fitness, ecological impacts may lead to physiologic consequences that reduce fitness for the environment to which a group had adapted. Dietary alterations can promote rapid changes in gut microbiota, but the roles of habituation to these diets and adaptations that occur across generations are unclear. ${ }^{25}$ As noted, traditional foods of Aboriginal peoples differed from those of European cultures (although there were likely commonalities with circumpolar countries), resulting in variations of microbiota. However, diminished access to traditional hunting grounds and food sources, together with more recent climate change impacts on wildlife migration patterns, fish stocks and plant habitats as well as the increased migration to urban settings have all resulted in substantial and relatively rapid dietary changes. With these rapid changes, particularly if they alter nutritional health early in life, pronounced consequences can emerge to favour the development of metabolic diseases (e.g., diabetes and heart disease, which are so common among Aboriginal peoples in Canada), ${ }^{26}$ as well as mental health issues. ${ }^{27}$

\section{Realizing a personalized treatment approach}

Research focused on developing novel personalized strategies is not as straightforward as simply adding another set of variables, particularly as cultural processes are more complex and dynamic than is often recognized (i.e., not simply a matter of ethnic categorization). Given the evolving aspects of culture, including changes across generations, a case could be made for simply focusing on the direct implications of culture for treatment strategies, without being concerned with whether and how culture affects biological processes. This caveat notwithstanding, defining the links between biological variations (e.g., epigenetic changes, polymorphisms) and the mental health of Aboriginal peoples in the context of cultural identity, climate change, activities and diet, as well as urbanization, might prove fundamental to realizing a personalized treatment approach.

Recognizing that culture entails more than mapping biological variations onto risks and mental health outcomes would also benefit from consideration of the links to culturally defined strengths. Even in this regard, it is important to appreciate that the strengths that contribute to individual hardiness might be unique to the changing social and environmental contexts and that there will be appreciable variation among Aboriginal peoples across Canada, and indeed around the world.

Acknowledgements: The authors thank Professor Patricia Boksa for many helpful comments on an earlier version of this editorial.

Affiliations: The Royal Ottawa Institute of Mental Health Research and Department of Neuroscience, Carleton University, Ottawa, Ont., Canada (Matheson); the Department of Psychiatry and School of Nursing, Dalhousie University, Halifax, NS, Canada (Bombay); and the Department of Neuroscience, Carleton University, Ottawa, Ont., Canada (Anisman).

Competing interests: None declared.

\section{References}

1. Khoury MJ, Iademarco MF, Riley WT. Precision public health for the era of precision medicine. Am J Prev Med 2016;50:398-401.

2. Insel $\mathrm{T}$, Cuthbert $\mathrm{B}$, Garvey $\mathrm{M}$, et al. Research domain criteria (RDoC): toward a new classification framework for research on mental disorders. Am J Psychiatry 2010;167:748-51.

3. Lilienfeld SO. The Research Domain Criteria. (RDoC): an analysis of methodological and conceptual challenges. Behav Res Ther 2014;62:129-39.

4. Khoury MJ, Galea S. Will precision medicine improve population health? JAMA 2016; 316:1357-8.

5. Kirmayer LJ, Craffa D. What kind of science for psychiatry. Front Hum Neurosci 2014;8:435.

6. Haene L, Grietens H, Verschueren K. From symptom to context: a review of the literature on refugee children's mental health. Hell J Psychol 2007;4:233-56.

7. Steel Z, Chey T, Silove D, et al. Association of torture and other potentially traumatic events with mental health outcomes among populations exposed to mass conflict and displacement: a systematic review and meta-analysis. JAMA 2009;302:537-49.

8. Kirmayer LJ. Cultural competence and evidence-based practice in mental health: epistemic communities and the politics of pluralism. Soc Sci Med 2012;75:249-56.

9. Sonne C, Carlsson J, Bech P, et al. Pharmacological treatment of refugees with trauma-related disorders: What do we know today? Transcult Psychiatry 2017;54:260-80.

10. Kirmayer LJ, Pedersen D. Toward a new architecture for global mental health. Transcult Psychiatry 2014;51:759-76. 
11. Boksa P, Joober R, Kirmayer LJ. Mental wellness in Canada's Aboriginal communities: striving toward reconciliation. J Psychiatry Neurosci 2015;40:363-6.

12. Hatala A, Desjardins $\mathrm{M}$, Bombay A. Reframing narratives of $\mathrm{Ab}$ original health inequity: exploring Cree Elder resilience and wellbeing in the contexts of historical trauma. Qual Health Res 2016;26:1911-27.

13. Wexler L. Intergenerational dialogue exchange and action: introducing a community-based participatory approach to connect youth, adults and elders in an Alaskan Native community. Int J Qual Methods 2011;10:248-64.

14. Ostapchuk J, Harper S, Willox AC, et al. Exploring Elders' and seniors' perceptions of how climate change is impacting health and well-being in Rigolet, Nunatsiavut. Inter J Indigen Health 2012;9:6-24.

15. Bourque F, Cunsolo Willox A. Climate change: The next challenge for public mental health? Int Rev Psychiatry 2014;26:415-22.

16. Marshall M, Marshall A, Bartlett C. Two-eyed seeing in medicine. In: Greenwood M, de Leeuw S, Lindsay NM, Reading C, editors. Determinants of Indigenous peoples' health in Canada. Beyond the social. Toronto (ON): Canadian Scholars Press; 2015. p. 16-24.

17. Kim HS, Sherman DK, Sasaki JY, et al. Culture, distress and oxytocin receptor polymorphism (OXTR) interact to influence emotional support seeking. Proc Natl Acad Sci U S A 2010;107:15717-157.

18. Talhelm T, Zhang X, Oishi S, et al. Large-scale psychological differences within China explained by rice versus wheat agriculture. Science 2014;344:603-8.
19. Yehuda R, Daskalakis NP, Bierer LM, et al. Holocaust exposure induced intergenerational effects on FKBP5 methylation. Biol Psychiatry 2016;80:372-80.

20. Bombay A, Matheson $\mathrm{K}$, Anisman $\mathrm{H}$. The intergenerational effects of Indian residential schools: implications for the concept of historical trauma. Transcult Psychiatry 2014;51:320-38.

21. Giri AK, Bharadwaj S, Banerjee P, et al. DNA methylation profiling reveals the presence of population-specific signatures correlating with phenotypic characteristics. Mol Genet Genomics 2017;7:655-62.

22. Nicoglou A, Merlin F. Epigenetics: a way to bridge the gap between biological fields. Stud Hist Philos Biol Biomed Sci. 2017; pii: S13698486(17)30044-4

23. Kelly JR, Minuto C, Cryan JF, et al. Cross talk: the microbiota and neurodevelopmental disorders. Front Neurosci 2017;11:490.

24. Fumagalli M, Moltke I, Grarup N, et al. Greenlandic Inuit show genetic signatures of diet and climate adaptation. Science 2015; 349:1343-7.

25. Morton ER, Lynch J, Froment A, et al. Variation in rural African gut microbiota is strongly correlated with colonization by Entamoeba and subsistence. PLoS Genet 2015;30:11:e1005658.

26. Block T, El-Osta A. Epigenetic programming, early life nutrition and the risk of metabolic disease. Atherosclerosis 2017;266:31-40.

27. Alam R, Abdolmaleky HM, Zhou JR. Microbiome, inflammation, epigenetic alterations, and mental diseases. Am J Med Genet B Neuropsychiatr Genet 2017;174:651-60

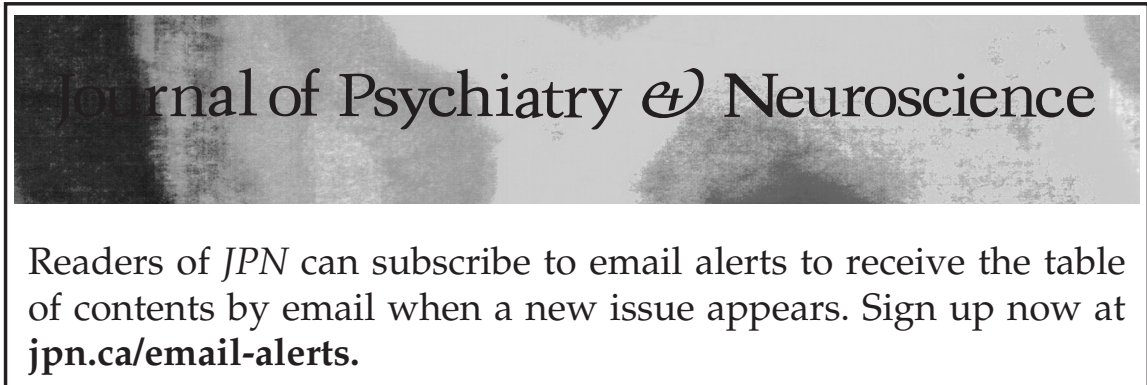

